\title{
Online test of the FRS Ion Catcher at GSI
}

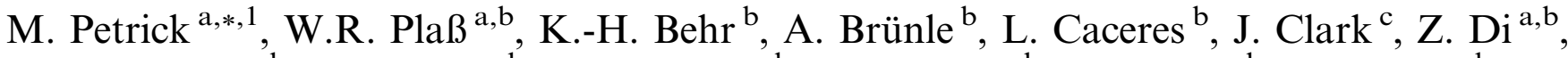 \\ S. Elisseev ${ }^{b}$, M. Facina ${ }^{d}$, A. Fettouhi ${ }^{a, b}$, H. Geissel ${ }^{a, b}$, W. Hüller ${ }^{b}$, M. Huyse ${ }^{d}$, \\ C. Karagiannis ${ }^{\text {b }}$, B. Kindler ${ }^{\text {b }}$, R. Knöbel ${ }^{\text {b }}$, Y. Kudryavtsev ${ }^{\text {d }}$, J. Kurcewicz ${ }^{\text {b }}$, T. Levant ${ }^{\text {c }}$, \\ Yu.A. Litvinov $^{\text {b }}$, B. Lommel ${ }^{\text {b }}$, M. Maier ${ }^{\text {b }}$, D.J. Morrissey ${ }^{\mathrm{e}}$, G. Münzenberg ${ }^{\mathrm{b}}$, M. Portillo ${ }^{\mathrm{e}}$, \\ G. Savard $^{\mathrm{c}}$, C. Scheidenberger ${ }^{\mathrm{a}, \mathrm{b}}$, P. Van Duppen ${ }^{\mathrm{d}}$, H. Weick $^{\mathrm{b}}$, M. Winkler ${ }^{\mathrm{b}}$, \\ B. Zabransky ${ }^{\mathrm{c}}$, for the FRS Ion Catcher Collaboration \\ a II. Physikalisches Institut, Justus-Liebig-Universität, D-35392 Gießen, Germany \\ ${ }^{\mathrm{b}}$ Gesellschaft für Schwerionenforschung, GSI, D-64291 Darmstadt, Germany

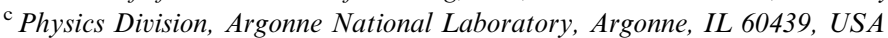 \\ ${ }^{\mathrm{d}}$ Katholieke Universiteit Leuven, B-3001 Leuven, Belgium \\ ${ }^{\mathrm{e}}$ National Superconducting Cyclotron Laboratory, Michigan State University, East Lansing, MI 48824, United States
}

Available online 12 July 2008

\begin{abstract}
At the FRS Ion Catcher at GSI, relativistic exotic ions produced by projectile fragmentation/fission are range-focused, slowed down and thermalised in a gas-filled stopping cell, extracted and made available to high-precision experiments with ions almost at rest. It is a prototype for a gas cell system at the Low-Energy Branch of the Super-FRS at FAIR. In an online experiment, the FRS Ion Catcher was commissioned successfully with relativistic nickel fragments. The overall efficiency of the system was measured as $(1.8 \pm 0.3) \%$ and can be divided into a stopping efficiency of $(5.0 \pm 1.1) \%$ and an extraction and transport efficiency of $(35.8 \pm 9.4) \%$. The overall efficiency is hence limited mostly by the stopping efficiency, which could be increased in the future by operating at higher gas cell pressures. From extraction time measurements of polyatomic ions formed in the gas cell extraction times of atomic ions of 20-50 ms can be derived. The potential of the system was illustrated by the half-life measurement of ${ }^{54} \mathrm{Co}$ with a short half-life of $193 \mathrm{~ms}$ only.
\end{abstract}

(c) 2008 Elsevier B.V. All rights reserved.

PACS: 29.25.Rm; 29.27.Ac; 29.38.Db

Keywords: Gas-filled stopping cell; Range focusing; Extraction time; Projectile fragmentation; Exotic nuclei

\section{Introduction}

At in-flight facilities, low-energy beams of exotic nuclei can be produced by slowing-down and thermalization of in-flight separated secondary beams in gas-filled stopping cells and subsequent extraction. The method combines the advantages of the in-flight and ISOL techniques [1] of

\footnotetext{
* Corresponding author.

E-mail address:

(M. Petrick).

${ }^{1}$ Part of Ph.D. thesis.
}

high selectivity, element-independence and short production time $(\sim 10 \mathrm{~ms})$, as well as of low kinetic energy (a few $10 \mathrm{keV}$ ) and small emittance (a few $\pi \mathrm{mm} \mathrm{mrad}$ ) of post-accelerated beams. It thus enables high-precision experiments with trapped or re-accelerated very short-lived nuclei, e.g. for nuclear structure studies, nuclear astrophysics, and the search for possible shortcomings of the standard model.

Gas-filled stopping cells are thus key elements of future low-energy ion beam facilities for exotic nuclei. At the FRS Ion Catcher facility at GSI [2], exotic nuclei produced and separated in-flight at the fragment separator FRS, are 
stopped in such a gas cell, which was designed and constructed at Argonne National Laboratory, extracted and made available to low-energy experiments. The FRS Ion Catcher is a prototype for a gas cell system at the LowEnergy Branch of the Super-FRS [3] at FAIR. It was commissioned in a first online experiment, giving for the first time a proof-of-principle with relativistic exotic nuclei. In addition, key performance parameters such as the stopping and extraction efficiency and extraction times were determined.

\section{Experimental setup}

Fig. 1 shows the setup used for the experiment. A $300 \mathrm{MeV} / \mathrm{u}^{58} \mathrm{Ni}$ primary beam was transported through the first half of the FRS and fragmented in a $2.5 \mathrm{~g} / \mathrm{cm}^{2}$ $\mathrm{Al}$ production target in the midplane of the FRS. The projectile fragments were dispersed according to their momentum in the second half of the FRS and energy-focused [4] using a wedge-shaped degrader system at the exit of the FRS. The selection of fragments with $A / Z=2$ led to almost monoenergetic secondary beams with almost identical mean velocities (corresponding to $\simeq 260 \mathrm{MeV} / \mathrm{u}$ ) at the exit of the FRS. The various fragments with $N=Z\left({ }^{56} \mathrm{Ni}\right.$, ${ }^{54} \mathrm{Co}$, etc.) have different mean ranges since for high-energy ions of equal velocity the range scales approximately like $R \propto A / Z^{2}$. Due to the energy focusing, the range differences exceed the width of the range straggling. This imposes a spatial (longitudinal) separation and thus, varying the degrader thickness, allows to stop an isotopically pure beam in the gas cell.

The center thickness of the degrader system is varied using the homogeneous degrader, which consists of two glass wedges. The wedge angle of the monoenergetic degrader is determined by adjusting the angle of two widdershins rotatable degrader discs. Using the range focusing achiev- able via the monoenergetic disc degrader [5], a minimum range distribution for the stopped ${ }^{54} \mathrm{Co}$ beam inside the gas cell of $15 \mathrm{mg} / \mathrm{cm}^{2}$ glass equivalent was achieved (Fig. 2).

Ion rates were measured in a multiple sampling ionisation chamber (MUSIC), while three scintillators in front of the gas cell determined the vertical distribution of the ions and thus gave the information about the ratio of ions entering the gas cell through the entrance window that had the same size as the center scintillator. A $100 \mu \mathrm{m}$ Ti foil was used as entrance window covering $250 \mathrm{~mm}$ in the horizontal (ion-optical dispersive) and $30 \mathrm{~mm}$ in the vertical direction.

The gas cell, developed at Argonne National Laboratory [7], is the main component of the experimental setup. It has a length of $1.25 \mathrm{~m}$ and was operated at a helium pres-

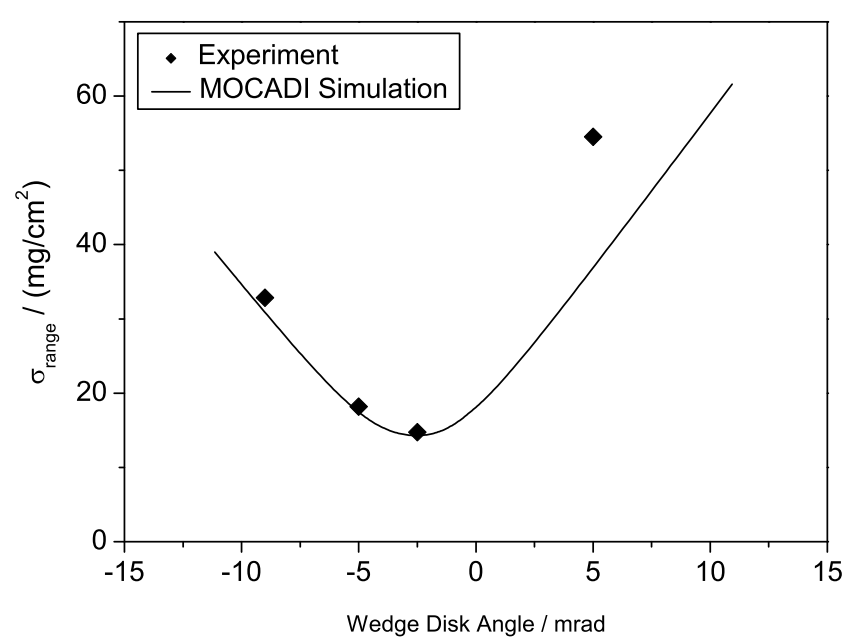

Fig. 2. Measured widths of the range distribution (standard deviation) in dependence of the angle of the monoenergetic disc degrader. A simulation with the program MOCADI [6] shows the good agreement with the measured data.

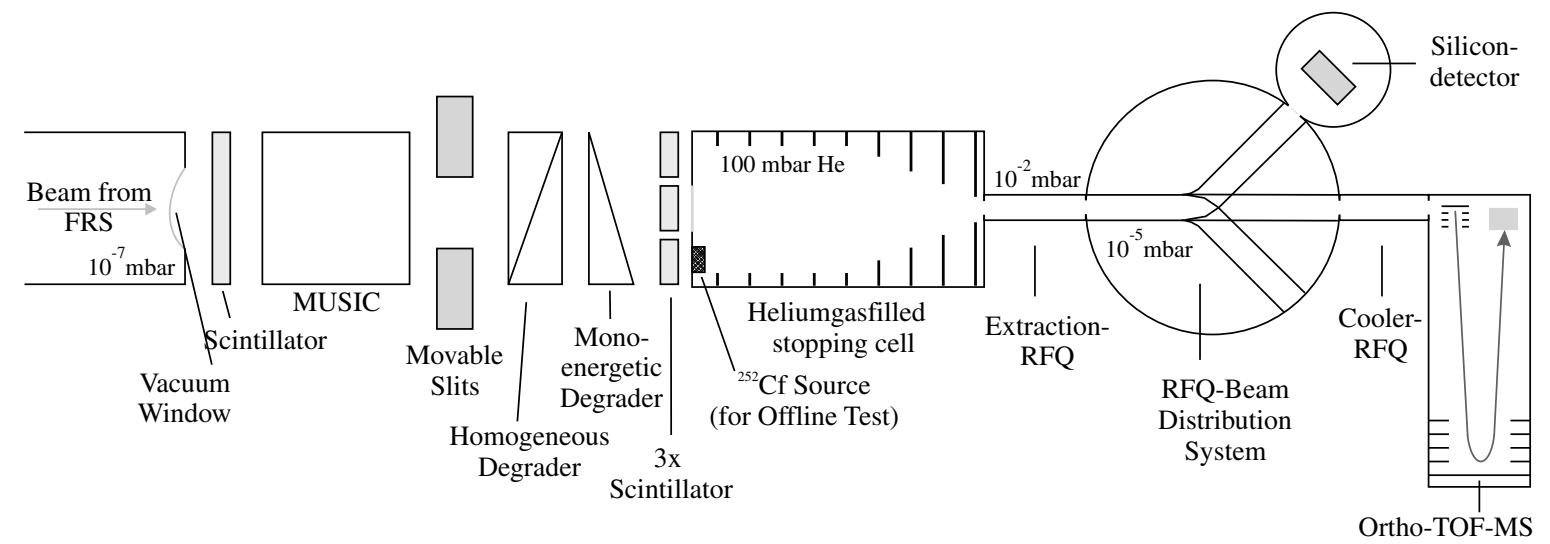

Fig. 1. Schematic figure of the experimental setup of the Ion Catcher experiment behind the FRS. A multi-wire proportional chamber (MWPC) is used for beam tracking and ion rates are measured in a multiple sampling ionisation chamber (MUSIC). The ions are range-focused in the degrader system, consisting of a homogeneous and a monoenergetic degrader. Three scintillators in front of the gas cell are employed to measure the ratio of ions that do not enter the gas cell. Ions are stopped in the gas cell in helium at a pressure of 100 mbar and extracted, separated from the gas in an extraction RFQ, and guided in an RFQ-based beam distribution system alternatively to a silicon detector for counting radioactive ions or a time-of-flight mass spectrometer with orthogonal acceleration (Ortho-TOF-MS) for identification of the ions and extraction time measurement. 
sure of $100 \mathrm{mbar}$, corresponding to a thickness of $2.3 \mathrm{mg} /$ $\mathrm{cm}^{2}$. Different DC- and RF-electrodes guide the stopped and thermalized ions to the nozzle, while repelling them from the gas cell walls. Extraction field strengths of $3.5 \mathrm{~V} / \mathrm{cm}$ up to $7 \mathrm{~V} / \mathrm{cm}$ were applied and an RF amplitude of $145 \mathrm{~V}$ at a frequency of $900 \mathrm{kHz}$ was used in the funnel section of the gas cell. The ions are swept out of the gas cell through the nozzle by the gas flow and separated from the helium gas in an extraction radiofrequency quadrupole (RFQ). The setup was pumped by a large roots-blower pump with a pumping speed of $5000 \mathrm{~m}^{3} / \mathrm{h}$ which is not oil-free. With a pressure of $100 \mathrm{mbar}$ inside the gas cell and a nozzle diameter of $1.6 \mathrm{~mm}$ a pressure of about $10^{-2}$ mbar is reached in the extraction RFQ. The setup currently cannot be baked.

Behind the extraction RFQ, the ions were guided with a novel RFQ-based ion beam distribution system [8] to a silicon detector or alternatively a time-of-flight mass spectrometer with orthogonal acceleration (Ortho-TOF-MS) [9]. The silicon detector served for counting of the $\beta$-decays of the extracted radioactive ions. The Ortho-TOF-MS is a broadband mass spectrometer with a mass resolving power of $10^{4}$ and thus allows for the detection and identification of all ions, including contaminant ions formed in the gas cell. Because of its high cycle frequency of $10 \mathrm{kHz}$, it could also be used to measure the extraction time profile of the ions out off the gas cell. Here, the heavy ion synchrotron SIS that delivered the primary beam was operated in a faster extraction mode with a spill length of $20 \mathrm{~ms}$, and the data acquisition of the Ortho-TOF-MS was triggered with the extraction-pulse from the SIS. Thus time-resolved mass spectra could be obtained.

The FRS Ion Catcher was developed, constructed and tested off-line with a ${ }^{252} \mathrm{Cf}$-source. The whole setup was transported to the final focus of the FRS and assembled there within three days before the start of the online test. All components were placed on air behind the FRS vacuum window. A leakage in the gas cell occurred during the setup, which could not be fixed because of the limited setup time allotted to the experiment.

\section{Results and discussion}

The most important result of the experiment is the fact that relativistic exotic nuclei can be stopped in and extracted from the gas cell efficiently. Due to the leak mentioned above and the not oil-free environment, a strong contamination of ions from water clusters and hydrocarbons formed in the gas cell was observed with the OrthoTOF-MS.

The Ortho-TOF-MS was also used to measure for the first time mass-resolved extraction times of the FRS IonCatcher. Extraction times are a crucial performance characteristic of gas-filled stopping cells, since they determine the minimum lifetime of the nuclei that can be investigated at the facility. Fig. 3 shows temporal mass-resolved extraction profiles of the contaminant ions. Note that the data

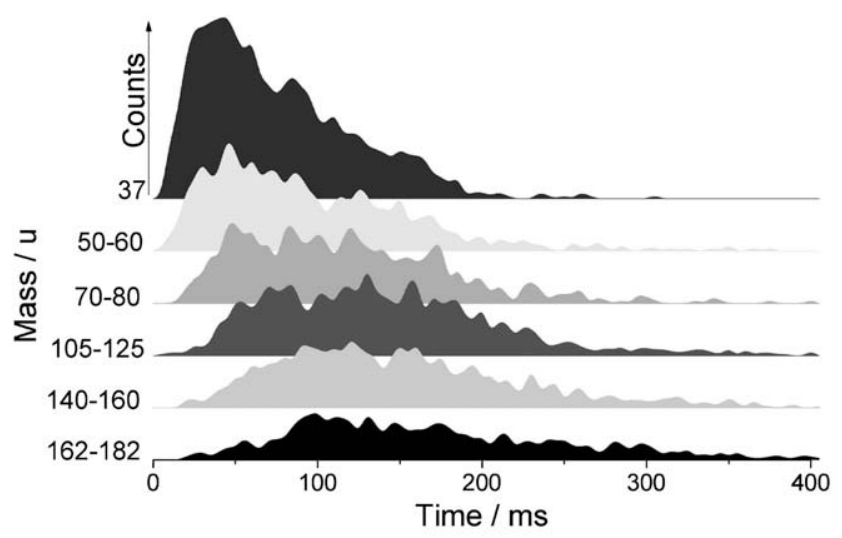

Fig. 3. Temporal mass-resolved extraction profiles of ions formed from water clusters and hydrocarbons extracted from the FRS Ion Catcher for an extraction field strength of $7 \mathrm{~V} / \mathrm{cm}$. The ion intensities are shown in dependence of the time between extraction-pulse from the heavy ion synchrotron and the time of detection in the Ortho-TOF-MS for different mass ranges.

for the different masses were acquired simultaneously without the need for a mass scan. Clearly, the extraction times increase with increasing mass. This dependence is summarized in Fig. 4 for two different extraction field strengths. From the mass-resolved extraction times for hydrocarbon ions, the extraction times for monoatomic ions can be estimated. Standard ion mobilities for monoatomic ions are almost mass-independent, and since hydrocarbon ions in the mass range around $100 \mu$ have ion mobilities that are smaller by a factor of $2-10$ than those of monoatomic ions [10-12], extraction times for monoatomic ions of $20-50 \mathrm{~ms}$ are derived at 100 mbar helium in the gas cell. This result is in good agreement with the calculation of the drift time of an ion with a mobility of $18 \times 10^{-4} \mathrm{~m}^{2} /(\mathrm{V} \mathrm{s})$ over a dis-

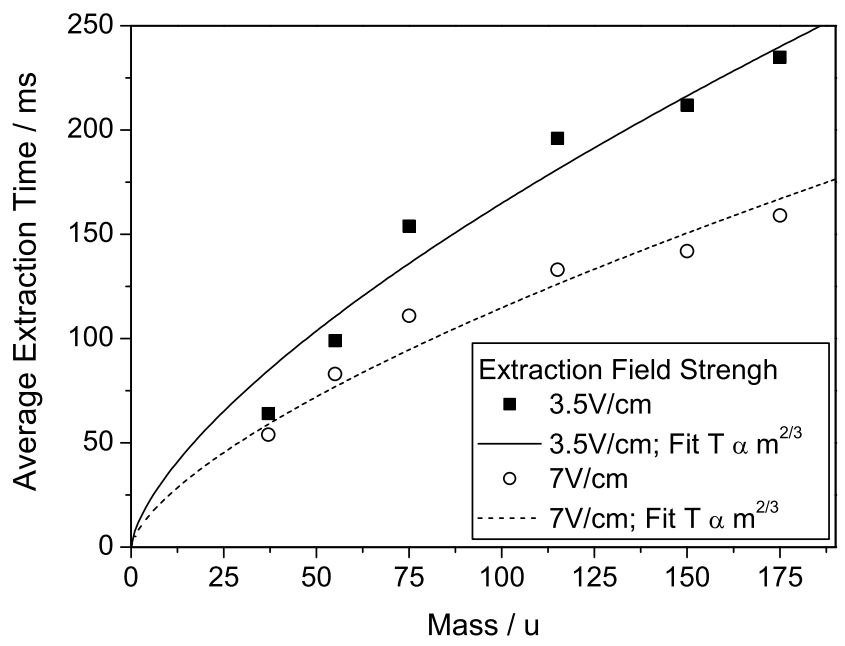

Fig. 4. Average extraction times of ions from the FRS Ion-Catcher in dependence of the ion mass for two different extraction field strengths. The fit $T \propto m^{2 / 3}$ assumes a simple ion mobility model with constant molecular density [10]. From the extraction times for the organic ions an extraction time for atomic ions of a few $10 \mathrm{~ms}$ can be estimated (see text). 
tance of $0.5 \mathrm{~m}$ in a pressure of $100 \mathrm{mbar}$ at a temperature of $300 \mathrm{~K}$, which amounts to $36 \mathrm{~ms}$.

Typical rates of ions entering the gas cell were $\sim 2 \times 10^{5}$ ions per spill extracted from the SIS within $\sim 8 \mathrm{~s}$. By measuring the ratio of the ion count rates of ${ }^{54} \mathrm{Co}$ ions the MUSIC in front of the gas cell to the count rate of $\beta$-decays on the silicon detector behind the RFQ system, the overall transmission efficiency of the FRS Ion Catcher can be determined. Overall an efficiency of $(1.8 \pm 0.3) \%$ was obtained. This number takes into account a few correction factors, which can be reliably estimated using the MonteCarlo simulation program MOCADI [6]. The beam spot in transverse, vertical and horizontal direction was calculated and the geometry of the setup was implemented (apertures, slits, dimensions of entrance window). Additionally, the number of ${ }^{54} \mathrm{Co}$ ions lost in secondary reactions in the degrader system and the entrance window of the gas cell were estimated and subtracted from the rate measured in the MUSIC. Furthermore, the stopping efficiency for ${ }^{54} \mathrm{Co}$ ions was determined from the measured range distributions (Fig. 2). Since the degrader thickness was adjusted such that a maximum yield was obtained, one can assume that the overlap of stopping volume $\left(\simeq 2.3 \mathrm{mg} / \mathrm{cm}^{2}\right)$ and range distribution $\left(\simeq 15 \mathrm{mg} / \mathrm{cm}^{2}\right)$ was maximized, which allows to determine the fraction of ions which come to rest inside the gas cell. Because of the finite lifetime of ${ }^{54} \mathrm{Co}$ and the extraction times of $20-50 \mathrm{~ms}$, the losses due to the decay of ${ }^{54} \mathrm{Co}$ before reaching the silicon detector were considered. Not only ${ }^{54} \mathrm{Co}$ reached the silicon detector, but a small contamination of ${ }^{55} \mathrm{Co}(2.4 \%)$ had to be subtracted as determined by calculations. In addition, the count rate on the silicon detector was corrected for its active solid angle for decay of ${ }^{54} \mathrm{Co}$ on the stopper foil in front of the detector.

In order to determine the extraction efficiency of the gas cell, one may divide the measured overall efficiency into a stopping, an extraction, and a transport efficiency. To convert the range data from the degrader material (glass) to helium gas the ratio of the stopping powers was converted using simulations with the computer programs SRIM and ATIMA. The results of both programs are in quite good agreement and were used for the stopping efficiency calculation. The stopping efficiency thus derived amounts to $(5.0 \pm 1.1) \%$. The extraction efficiency is given by the ratio of overall efficiency and stopping efficiency and is $(35.8 \pm 9.4) \%$. This value also includes the transport efficiency from the exit gas cell through the RFQ system to the detector, which is estimated as $(75 \pm 25) \%$.

The overall efficiency is hence limited mostly by the stopping efficiency, which could be increased in the future by operating at higher gas cell pressures. Also a higher ion-optical resolution will allow stronger energy-focusing and thus increased stopping efficiency. In general the situation becomes more favourable for heavier ions, where range and range straggling are smaller.

Finally, the successful commissioning of the FRS Ion Catcher is illustrated by the lifetime measurement of the ground state and the isomeric state of ${ }^{54} \mathrm{Co}$. The measured half-lives are $(198 \pm 4) \mathrm{ms}$ and $(106 \pm 20) \mathrm{s}$, respectively, are in good agreement with the literature values of $(193.28 \pm 0.07) \mathrm{ms}$ and $(88.8 \pm 1.2) \mathrm{s}$ [13]. The measurement of the short half-life of the ground state, in particular, shows the potential of the system for future applications.

\section{Conclusion}

The FRS Ion Catcher was commissioned successfully in an online experiment. For the first time, relativistic exotic nuclei were range-focused, stopped in a gas cell, and extracted. The overall efficiency amounts to $(1.8 \pm 0.3) \%$ and can be divided into a stopping efficiency of $(5.0 \pm 1.1) \%$ and an extraction and transport efficiency of $(35.8 \pm 9.4) \%$. The overall efficiency is hence limited mostly by the stopping efficiency, which could be increased in the future by operating at higher gas cell pressures. From extraction time measurements of polyatomic ions formed in the gas cell extraction times of atomic ions of 20 $50 \mathrm{~ms}$ can be derived. The potential of the system was illustrated by the half-life measurement of ${ }^{54} \mathrm{Co}$ with a short half-life of $193 \mathrm{~ms}$ only.

The FRS Ion Catcher is a prototype for a gas cell system at the Low-Energy Branch of the Super-FRS at FAIR. Here, in-flight separated exotic nuclear beams from the Super-FRS will be converted to low-energy beams for the precision experiments MATS [14] (advanced trapping setup for accurate mass measurements), LASPEC [15] (laser spectroscopy to determine nuclear moments) and exo $+p-$ bar [16] (to explore the nuclear periphery of trapped exotic nuclei by anti-proton reactions).

\section{Acknowledgement}

This work was supported by the European Community under contract number 515873 (DIRACsecondary-Beams) and the Helmholtz Association and GSI under contract number VH-NG-033.

\section{References}

[1] H. Geissel, G. Münzenberg, K. Riisager, Ann. Rev. Nucl. Part Sci. 45 (2005) 163

[2] M. Maier et al., GSI Scientific Report 2005 (2006) p. 41.

[3] H. Geissel et al., Nucl. Instr. and Meth. B 204 (2003) 71.

[4] C. Scheidenberger et al., Nucl. Instr. and Meth. B 204 (2003) 119.

[5] M. Maier, Ph.D. thesis, Justus-Liebig-Universität Gießen, 2004.

[6] N. Iwasa, Nucl. Instr. and Meth. B 126 (1997) 284.

[7] G. Savard et al., Nucl. Instr. and Meth. B 204 (2003) 582.

[8] M. Petrick, W.R. Plaß, H. Geissel, M. Maier, C. Scheidenberger, GSI Scientific Report 2004 (2005) p. 330

[9] S. Eliseev, Ph.D. Thesis, Justus-Liebig-Universität Gießen, 2004.

[10] H.E. Revercomb, E.A. Mason, Anal. Chem. 47 (1975) 970.

[11] L.A. Viehland, E.A. Mason, At. Data Nucl. Data Tables 60 (1995) 37. 
[12] S.J. Valentine, A.E. Counterman, D.E. Clemmer, J. Am. Soc. Mass Spectrom. 10 (1999) 1188.

[13] Huo Junde, Huo Su, Nuclear Data Sheets 107 (2006) 1393.

[14] K. Blaum et al., Technical Report for MATS, 2005. http:// www.gsi.de/fair/experiments/NUSTAR/.
[15] P. Champbell et al., Technical Report for LASPEC, 2005. http:// www.gsi.de/fair/experiments/NUSTAR/.

[16] M. Wada et al., Technical Report for exo+pbar, 2005. http:// www.gsi.de/fair/experiments/NUSTAR/. 